\title{
Entangled Absorption of a Single Photon with a Single Spin in Diamond
}

\author{
Hideo Kosaka* and Naeko Niikura \\ Yokohama National University, 79-5 Tokiwadai, Hodogaya, Yokohama 240-8501, Japan
}

(Received 2 October 2014; published 5 February 2015)

\begin{abstract}
Quantum entanglement, a key resource for quantum information science, is inherent in a solid. It has been recently shown that entanglement between a single optical photon and a single spin qubit in a solid is generated via spontaneous emission. However, entanglement generation by measurement is rather essential for quantum operations. We here show that the physics behind the entangled emission can be time reversed to demonstrate entangled absorption mediated by an inherent spin-orbit entanglement in a single nitrogen vacancy center in diamond. Optical arbitrary spin state preparation and complete spin state tomography reveal the fidelity of the entangled absorption to be $95 \%$. With the entangled emission and absorption of a photon, materials can be spontaneously entangled or swap their quantum state based on the quantum teleportation scheme.
\end{abstract}

DOI: 10.1103/PhysRevLett.114.053603

PACS numbers: 42.50.Ct, 03.65.Wj, 76.30.Mi, 78.47.jb

Generation of remote quantum entanglement is a central challenge in quantum information science [1]. The common way to generate entanglement relies on optical components such as a nonlinear crystal [2] or a beam splitter [3-10]. It has been recently shown that entanglement between a single optical photon and a single atom [11] or a single electron spin in a solid [12,13] can be generated via spontaneous emission. Among various kinds of solids, a nitrogen-vacancy (NV) center in diamond is expected to be an ideal solid-state quantum memory, since the spin coherence of an electron or nuclear spin can be maintained over a second order [14]. It has been demonstrated that those spins are coherently controlled with a microwave, radio wave $[15,16]$, or light wave [17-19] to generate and detect local entanglement between spins [20]. The entanglement inherent in a solid can be utilized to generate even remote entanglement. The entanglement in an electronic excited state in a NV center has been used to generate entanglement between an emitted optical photon and a stored electron spin [12]. This demonstration offered a simple and straightforward way to generate entanglement between flying and stationary qubits using merely a photon emission without the need for any coherent control, entangled photon source [2], or even a beam splitter [3]. Based on this emission-based entanglement generation, entanglement between two remote NV centers has been generated with the help of a beam splitter [10], which plays the crucial role of the entanglement measurement. In this work, we show that the beam splitter can be replaced by the NV center itself [Fig. 1(a)] by utilizing the inherent entanglement not only for entanglement generation but also for the entanglement measurement between a stored electron spin and an absorbed optical photon. With the entangled emission and absorption of a photon, materials can be entangled or swap their quantum state based on the quantum teleportation scheme [1], which is the core function of a solid-state quantum repeater needed for quantum networks as well as quantum computers [21-23].

The mechanism and technologies used in this demonstration are closely related to the coherent dark state or bright state projections introduced for all-optical control of a NV spin [17]. Instead of the asymmetric nondegenerate spin states used in the previous work, here we use symmetric degenerate spin states with angular momentum $m_{s}= \pm 1$ (hereafter denoted as $| \pm 1\rangle$ ) associated with zero projection of orbital angular momentum (denoted as $\left|E_{0}\right\rangle$ ) as our qubit basis states, offering a phase-frozen quantum memory when $| \pm 1\rangle$ are set to their complete degeneracy. In this extreme, the qubit state of an electron is naturally represented by a spin state vector in the Bloch sphere, as that of a photon is represented by a polarization state vector in the Poincaré sphere [Fig. 1(a)]. Although these two qubits cannot directly interact with each other, strong correlation is possible through the orbital state of an electron, which works as an ancilla qubit to mediate the entanglement. The related electronic states in the demonstration form a degenerate $V-\Lambda$ four-level system [Fig. 1(b)]. The degenerate spin sublevels $| \pm 1\rangle$ are coupled to the spin ground state with $m_{s}=0$ (denoted as $|0\rangle$ ) with a resonant microwave photon forming the $V$ system and to the orbital excited state $\left|A_{2}\right\rangle$ with a resonant optical photon forming the $\Lambda$ system. The $A_{2}$ state is the source of the entanglement measurement as it forms the $\left|\psi^{+}\right\rangle$state, which is one of the entangled states known as Bell states, with the help of the spin-orbit and spin-spin interaction as

$$
\left|A_{2}\right\rangle=\frac{1}{\sqrt{2}}\left(|+1\rangle\left|E_{-}\right\rangle+|-1\rangle\left|E_{+}\right\rangle\right),
$$

where $\left|E_{ \pm}\right\rangle$are orbital states with angular momentum \pm 1 along the NV axis [24-29].

The electron spin state is optically prepared and measured using the pulse sequence and experimental setup 

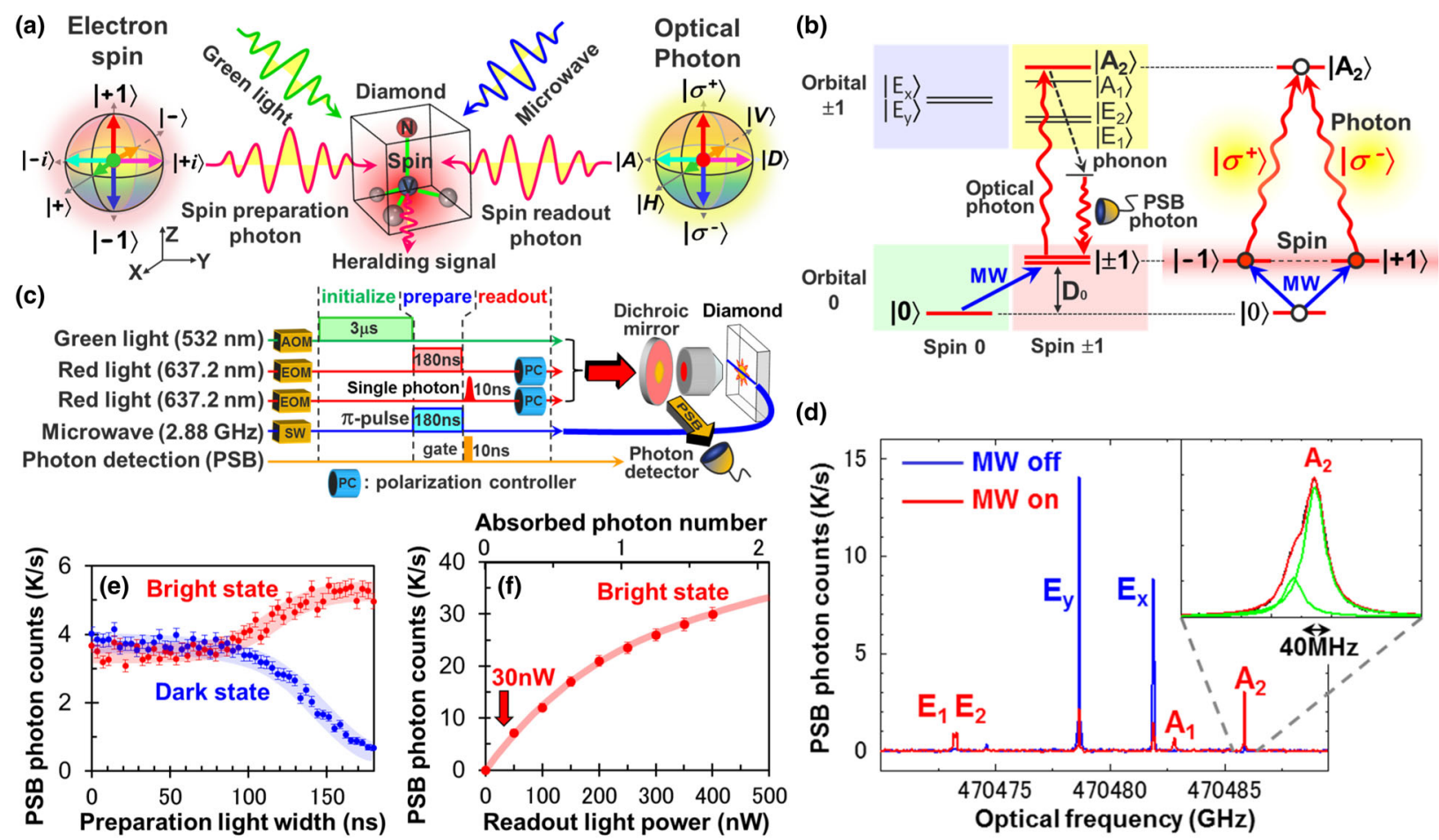

FIG. 1 (color online). (a) Scheme for the entanglement measurement. Quantum correlation between optically stored electron spin and incoming readout optical photon is measured via resonant absorption, which is heralded by a phonon sideband (PSB) photon detection. (b) Related energy levels of the NV center. Spin triplet sublevels $| \pm 1\rangle$ are used as spin qubit bases. (c) Pulse sequence and setup used for the experiments. AOM denotes acousto-optic modulator, EOM denotes electro-optic modulator, and SW denotes microwave switch (d) Photoluminescence excitation spectrum with (red) and without (blue) microwave resonant to $D_{0}$ shown in (b). Inset is a close-up view of the $A_{2}$ line, which is decomposed into two Lorentzian curves. (e) Photon count rate emitted in the PSB as a function of the pulse width of the preparation light to prepare the bright state in $|+i\rangle$ (red) and the dark state in $|-i\rangle$ (blue) against the readout light in $|D\rangle$. (f) Photon count rate as a function of the power of the readout light corresponding to the bright state. The curve is a fit to the data assuming the Poisson distribution where one photon is absorbed on average at $240 \mathrm{nW}$. The error bars represent $1 \sigma$ shot noise.

outlined in Fig. 1(c). We start from the $|0\rangle$ state by irradiating a green light $(532 \mathrm{~nm}, 100 \mu \mathrm{W})$ for $3 \mu \mathrm{s}$. The population is then transferred into the spin qubit basis states $| \pm 1\rangle$ by irradiating a microwave $\pi$ pulse $(2.88 \mathrm{GHz}$ $180 \mathrm{~ns}$ ) resonant to the zero-field spin splitting energy $D_{0}$. Synchronized with the microwave pulse, a red light $(637.2 \mathrm{~nm}, 480 \mathrm{nW})$ resonant to the $A_{2}$ state [Fig. 1(d)] is irradiated for $180 \mathrm{~ns}$ to prepare an arbitrary superposition through the absorption and emission of multiple optical photons [Fig. 1(e)]. The preparation is possible only with the $A_{2}$ state, since the others decay into $|0\rangle$. Just after the spin preparation, another red light $(637.2 \mathrm{~nm}, 30 \mathrm{nW})$ with arbitrary controlled polarization is irradiated for $10 \mathrm{~ns}$ to read out the spin state in the polarization-defined measurement basis. Synchronized with the readout light, a single photon detector is gated to count only the phonon sideband (PSB) photon using a bandpass filter passing through 650 to $750 \mathrm{~nm}$. Depending on the photon count rate, we can determine if the election spin is in a bright (high count rate) or dark state (low count rate). The readout power dependence of the PSB photon count for the bright state [Fig. 1(f)] indicates that the absorption probability of multiple photons $(0.7 \%$ at most) is much less than that of a single photon $(11 \%)$ at $30 \mathrm{nW}$ used in the measurement.
The preparation originates from the total angular momentum conservation in an optical transition in such a way that a photon with right- or left-handed circular polarization $\left|\sigma^{ \pm}\right\rangle$raises or lowers the orbital angular momentum from $E_{0}$ to $\left|E_{ \pm}\right\rangle$without changing the spin angular momentum. Since the $A_{2}$ state couples the $\left|E_{ \pm}\right\rangle$ orbital with the $|\mp 1\rangle$ spin as in Eq. (1), the $\left|\sigma^{ \pm}\right\rangle$photon excites the $|\mp 1\rangle$ spin to the $A_{2}$ state. Owing to the fixed coherence of the $A_{2}$ state, the correlation applies to any superposition states as the $\alpha\left|\sigma^{+}\right\rangle+\beta\left|\sigma^{-}\right\rangle$photon excites the $\alpha^{*}|-1\rangle+\beta^{*}|+1\rangle$ spin, which is called the bright state [17]. Note that the bright state is bit flipped from the photon state. The $\left|A_{2}\right\rangle$ state then spontaneously relaxes back to the $| \pm 1\rangle$ states with equal probabilities. If we maintain the irradiation, only the bright state component is excited until reaching the state orthogonal to the bright state, called the dark state $\alpha|+1\rangle-\beta|-1\rangle$ [17]. Note that the dark state is phase flipped from the photon state. This mechanism, referred to as a dark state preparation based on the coherent population trapping in the degenerate $\Lambda$ system, is used to prepare an arbitrary spin state.

The degenerate $\Lambda$ system also works as a measure of spin-photon entanglement. To prove the entanglement property of the absorption, we here show a strong 

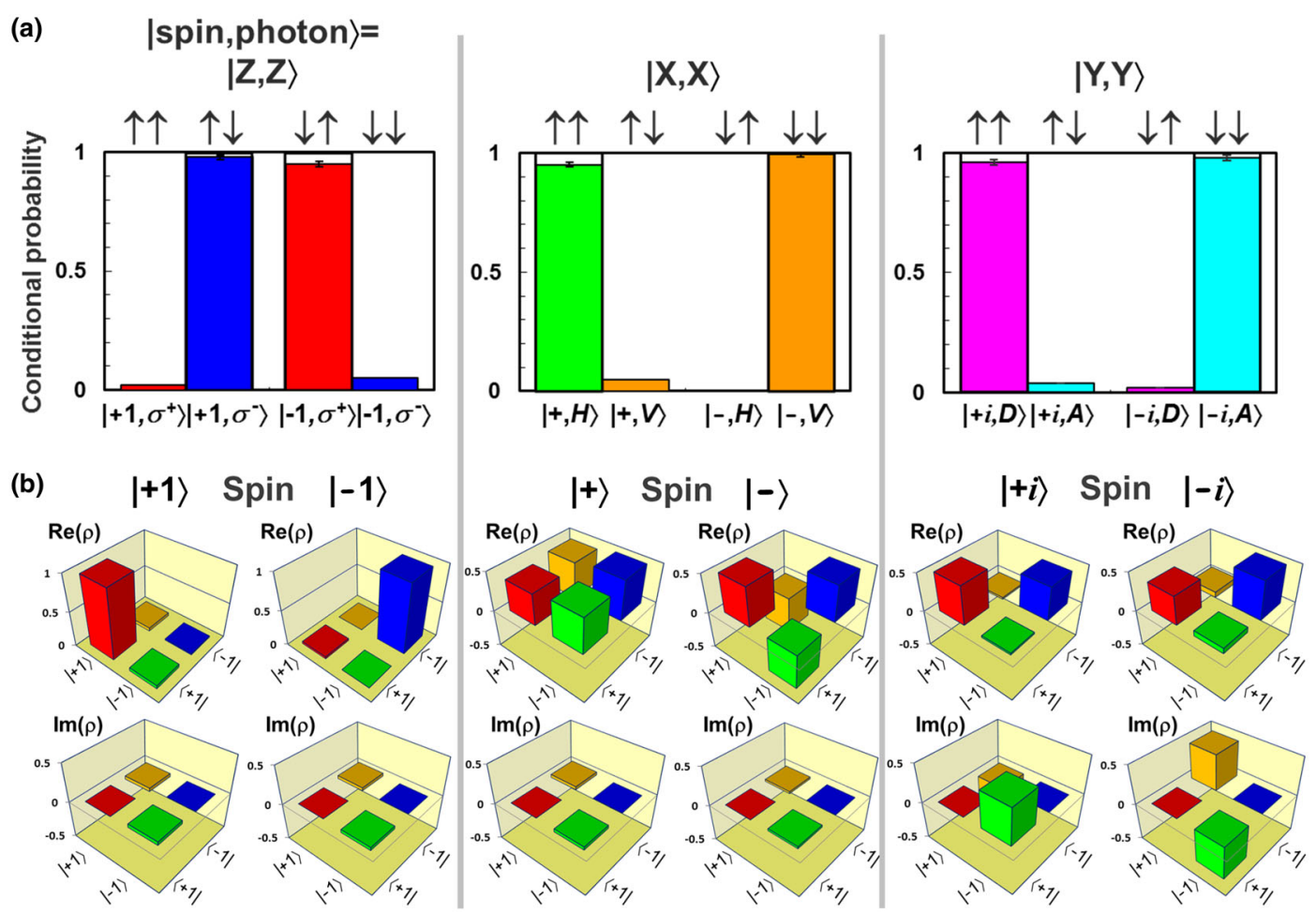

FIG. 2 (color online). (a) Conditional probabilities of detecting the PSB photon when the prepared spin and the readout photon are both along $Z, X$, and $Y$ bases (see text). Strong anticorrelation in $Z$ and correlations in $X$ and $Y$ reveal the entanglement measurement property of the absorption. State notations are defined in Fig. 1(a). (b) Density matrices of the spin states nominally prepared in $\pm Z$ $(| \pm 1\rangle), \pm X(| \pm\rangle)$, and $\pm Y(| \pm i\rangle)$ by the spin preparation photons, reconstructed by the spin state tomography technique. Here we use strong readout power $(200 \mathrm{nW})$ to reduce a readout error.

correlation between electron spin and photon polarization for arbitrary superposition states that correspond to the bright states. In this context, the entanglement measurement can be regarded as the bright state projection.

We use a native NV center in high-purity type-IIa chemical-vapor-deposition grown bulk diamond with a $\langle 001\rangle$ crystal orientation (electronic grade from Element Six) without any dose or annealing. The negatively charged $\mathrm{NV}$ center located at about $50 \mu \mathrm{m}$ below the surface is found using a confocal laser microscope. The narrow line width (40 MHz) of the $A_{2}$ state [Fig. 1(d)] indicates that the NV is almost free from spectral diffusion and charge fluctuation [27]. Although the main peak is used for the experiments, both components perform the same. A $25-\mu \mathrm{m}$ copper wire mechanically attached to the surface of the diamond is used to apply a microwave for the optically detected magnetic resonance measurement. The NV center used in the experiment does not show hyperfine splitting caused by ${ }^{13} \mathrm{C}$ nuclear spins within $0.1 \mathrm{MHz}$. Although the $\mathrm{NV}$ axis is off aligned to the optical axis, it does not have a major effect on the experimental results. The geomagnetic field projected onto the NV axis is compensated with a carefully positioned permanent magnet by monitoring the optically detected magnetic resonance spectrum within $0.1 \mathrm{MHz}$. The time dependences of Rabi oscillation and Ramsey interference are also used to fine tune the field. All experiments are performed at $5 \mathrm{~K}$ in a helium-free cryostat.
The excited-state orbital splitting between $m_{s}=0$ spin sublevels [ $E_{x}$ and $E_{y}$ in Fig. 1(b)] of the studied NV center is approximately $3.0 \mathrm{GHz}$, which implies a strain energy of $1.5 \mathrm{GHz}$ at the orbital excited state, independent of the cooling cycles. Spin-orbit coupling protects the $A_{2}$ state against the small strain to preserve the polarization properties of its optical transition [11,29].

Figure 2(a) shows the measured correlation between the prepared spin state and the readout photon state not only for the basis states defined by the optical axis $| \pm 1\rangle$ ( $Z$ basis) but also for the superposition states $| \pm\rangle=(1 / \sqrt{2})(|+1\rangle \pm|-1\rangle)$ ( $X$ basis) and $| \pm i\rangle=(1 / \sqrt{2})(|+1\rangle \pm i|-1\rangle)$ ( $Y$ basis). When both the spin and photon states are prepared along $Z$ (denoted as the $|Z, Z\rangle$ basis), the conditional probability of detecting a photon in $\left|\sigma^{ \pm}\right\rangle$after the detection of a $\sigma^{+}$or $\sigma^{-}$photon shows a strong anticorrelation with the prepared spin state in $| \pm\rangle$ (odd parity). The coherence of the joint measurement is revealed in superposition bases along the $|X, X\rangle$ basis and the $|Y, Y\rangle$ basis, which also show a strong correlation (even parity). The parity difference originates from the bit flip property of the bright state mentioned above.

The correlation measurement shown above assumes that the spin state is prepared as intended with the preparation photon. To quantitatively evaluate the prepared spin state, we apply the scheme of spin state tomography introduced in Ref. [19]. The reconstructed density matrices shown in 
(a)

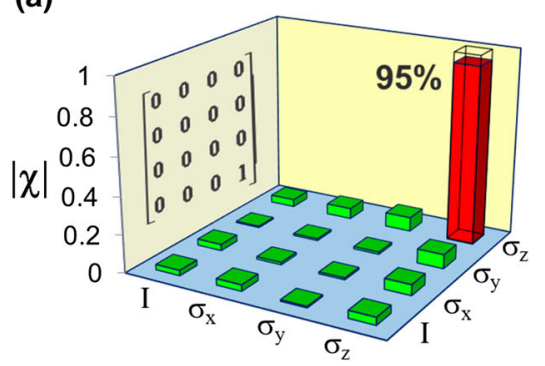

(b)

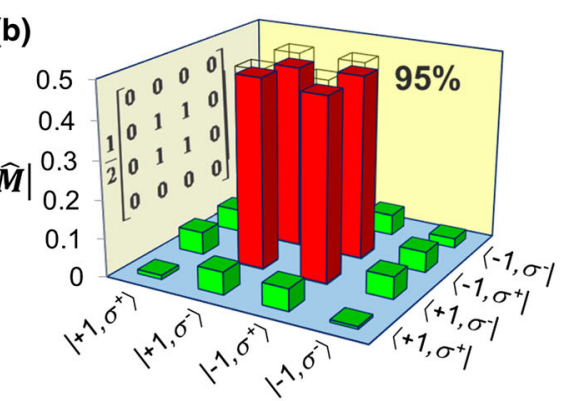

FIG. 3 (color online). (a) The $\chi$ matrix representation of the spin preparation process. The element shown by the red bar at $\left(\sigma_{z}, \sigma_{z}\right)$, which corresponds to the phase flip process originating from the relative phase of the dark state, is used for the estimation of the spin preparation fidelity to be $95 \%$. (b) The operator matrix that represents the joint measurement between the electron spin and the photon polarization. The off-diagonal elements show two-qubit coherence. The elements shown in the red bars, corresponding to the spinphoton entanglement originating from the spin-orbit entanglement in the $A_{2}$ state, are used for the estimation of the entanglement measurement fidelity to be $95 \%$. The ideal matrix elements are shown by the framed bars and insets.

Fig. 2(b) well coincide with the intended states. On the other hand, the polarization state of the preparation photon is well defined by using a polarimeter. From the complete knowledge of the states for both the preparation photon and the prepared spin, we can evaluate the spin preparation as a quantum process that maps the photon state $\hat{\rho}_{\text {photon }}$ to the spin state $\hat{\rho}_{\text {spin }}$ as the $\chi$ (chi) matrix representation $\hat{\rho}_{\text {spin }}=$ $\sum_{i, j} \chi_{i j} \sigma_{i} \hat{\rho}_{\text {photon }} \sigma_{j}$, where $\sigma_{i}$ denotes the Pauli operators $\sigma_{x}$, $\sigma_{y}, \sigma_{z}$, respectively, for $i=1,2,3$ and the identity operator $I$ for $i=0$ as a basis set of the quantum operation $[1,30]$. Figure 3(a) shows the obtained $\chi$ matrix. Note that the major element at $\left(\sigma_{z}, \sigma_{z}\right)$ corresponds to the phase flip operation $\hat{\rho}_{\text {spin }}=\sigma_{z} \hat{\rho}_{\text {photon }} \sigma_{z}$, which means the state vector of the prepared spin in the Bloch sphere is a $\pi$ rotation around the $z$ axis from the state vector of the preparation photon in the Poincare sphere. This operation originates from the phase flip property of the dark state mentioned above. The fidelity of the spin preparation process to the dark state is given by the real part of the $\left(\sigma_{z}, \sigma_{z}\right)$ component as $95 \%$.

Assuming perfect spin preparation, we can now tomographically reconstruct the measurement operator $\hat{M}$ upon the detection of the readout photon absorption with the complete knowledge of both the prepared spin and the readout photon. The matrix elements are calculated by solving linear equations to give the probability $P$ of projecting the given spin-photon joint state $\hat{\rho}_{\text {in }}$ onto the measurement operator $\hat{M}$ as $P=\operatorname{Tr}\left(\hat{M} \hat{\rho}_{\text {in }}\right)$ in all combinations of the $\{X, Y, Z\}$ bases, not only the $|X, X\rangle,|Y, Y\rangle$, and $|Z, Z\rangle$ bases used for the correlation measurement. The projection probability $P$ is set to half of the conditional probability shown in Fig. 2(a) based on the knowledge of the $A_{2}$ state given in Eq. (1). The reconstructed measurement operator $\hat{M}$ on the basis of the spin of the electron and the polarization of a photon shown in Fig. 3(b) indicates that the measurement projects the spin-photon joint state into their entangled state as

$$
\hat{M}=\frac{1}{2}\left(|+1\rangle\left|\sigma^{-}\right\rangle+|-1\rangle\left|\sigma^{+}\right\rangle\right)\left(\langle+1|\left\langle\sigma^{-}\right|+\langle-1|\left\langle\sigma^{+}\right|\right) .
$$

Note that this form coincides with the density operator $\hat{\rho}_{A_{2}}$ of the $A_{2}$ state on the basis of the spin and orbital of an electron assuming the ideal polarization selection rule. This coincidence implies that the projecting entangled state is defined by the $A_{2}$ state. The fidelity of the entanglement measurement is calculated as the probability $P$ by substituting the maximum entangled state for $\hat{\rho}_{\text {in }}$ to be $95 \%$. The possible reasons for the fidelity degradation include misalignment of the NV axis against the optical axis, the mixing of the $E_{1}$ and $E_{2}$ states into the $A_{2}$ state due to nonaxial crystal strain, and the hyperfine interaction between the electron and nuclear spins.

The fidelity of the entanglement measurement $F$ strongly depends on the powers of the preparation light $P$ (Fig. 4) as $V=1-2^{-P / P_{1}}$, where the visibility $V$ defines $F=(V+1) / 2$ and $P_{1}(210 \mathrm{nW})$ is the power at which a single cycle of photon absorption and emission occurs. The result indicates that a few cycles are enough to reach $F>95 \%$. On the other hand, the fidelity is nearly independent of the readout power since the bright state projection needs only one photon absorption. The slight increase in fidelity with the increase in the readout power is well fitted by considering the expectation time of the first photon absorption during $10 \mathrm{~ns}$ under the effect of ${ }^{14} \mathrm{~N}$ nuclear spin.

The entanglement measurement has been demonstrated via spin-photon coherent resonant absorption heralded by phonon sideband photon emission. We utilized the electron orbital qubit as an ancilla that is entangled with the spin qubit to measure otherwise noninteracting spin-photon entanglement. The other keys are techniques for arbitrary spin state preparation and complete spin state tomography in a completely degenerate $\Lambda$ system. The scheme can be applied to other systems such as other impurity centers in diamond, phosphorous donors in silicon, and rare-earthion-doped crystals. Although we did not prepare entangled states to prove the entanglement measurement, the fidelity estimation is mathematically as correct as that in the reverse case of entanglement generation. 


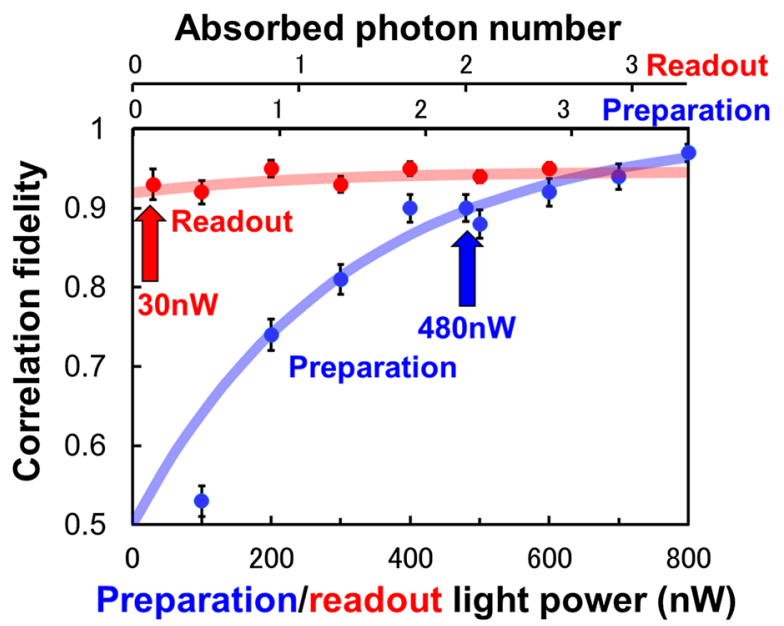

FIG. 4 (color online). The correlation measurement fidelity along $X(|+\rangle$ preparation and $|H\rangle$ readout), which gives the lower bound of the entanglement measurement fidelity, as functions of preparation (blue) and readout (red) light powers. The results indicate that the spin preparation needs multiple photons to project the spin into the dark state, whereas the spin readout needs only one photon to project the spin into the bright state. The fitted curves are explained in the text. Arrows indicate the powers used for the correlation and entanglement experiments. The error bars represent $1 \sigma$ shot noise.

The importance of the entangled absorption together with the entangled emission is that electrons can swap the remote entanglement by simply concatenating the emission and absorption processes in one way based on the quantum transmitter and receiver design. In contrast to the commonly used beam splitters, the entangled absorber does not require timing adjustment at all, thus drastically easing the system design. The configuration is suitable for a one-way architecture of quantum repeaters to build high bit-rate large-scale quantum communication networks $[21,22]$ and distributed quantum computers [23].

We thank Joerg Wrachtrup, Philipp Neumann, Petr Siyushev, Helmut Fedder, Sen Yang, Ali Momenzadeh Seyed, Norikazu Mizuochi, Kae Nemoto, William Munro, Scharfenberger Burkhard, and Keiichi Edamatsu for their discussions and experimental help. This work was supported by the NICT Quantum Repeater Project, by the FIRST Quantum Information Project, and by a Grant-inAid for Scientific Research (A)-JSPS (No. 24244044).

*kosaka@ynu.ac.jp

[1] M. A. Nielsen and I. L. Chuang, Quantum Computation and Quantum Information (Cambridge University Press, Cambridge, England, 2000).

[2] A. Aspect, P. Grangier, and G. Roger, Phys. Rev. Lett. 49, 91 (1982).

[3] C. K. Hong, Z. Y. Ou, and L. Mandel, Phys. Rev. Lett. 59, 2044 (1987).
[4] D. L. Moehring, P. Maunz, S. Olmschenk, K. C. Younge, D. N. Matsukevich, L.-M. Duan, and C. Monroe, Nature (London) 449, 68 (2007).

[5] R. B. Patel, A. J. Bennett, I. Farrer, C. A. Nicoll, D. A. Ritchie, and A. J. Shields, Nat. Photonics 4, 632 (2010).

[6] E. B. Flagg, A. Muller, S. V. Polyakov, A. Ling, A. Migdall, and G. S. Solomon, Phys. Rev. Lett. 104, 137401 (2010).

[7] H. Bernien, L. Childress, L. Robledo, M. Markham, D. Twitchen, and R. Hanson, Phys. Rev. Lett. 108, 043604 (2012).

[8] W. Pfaff, T. H. Taminiau, L. Robledo, H. Bernien, M. Markham, D. J. Twitchen, and R. Hanson, Nat. Phys. 9, 29 (2013).

[9] H. Bernien et al., Nature (London) 497, 86 (2013).

[10] W. Pfaff et al., Science 345, 532 (2014).

[11] B. B. Blinov, D. L. Moehring, L. M. Duan, and C. Monroe, Nature (London) 428, 153 (2004).

[12] E. Togan et al., Nature (London) 466, 730 (2010).

[13] K. De Greve et al., Nature (London) 491, 421 (2012).

[14] N. Bar-Gill, L. M. Pham, A. Jarmola, D. Budker, and R. L. Walsworth, Nat. Commun. 4, 1743 (2013).

[15] F. Jelezko, T. Gaebel, I. Popa, A. Gruber, and J. Wrachtrup, Phys. Rev. Lett. 92, 076401 (2004); F. Jelezko, T. Gaebel, I. Popa, M. Domhan, A. Gruber, and J. Wrachtrup, Phys. Rev. Lett. 93, 130501 (2004).

[16] M. V. G. Dutt, L. Childress, L. Jiang, E. Togan, J. Maze, F. Jelezko, A. S. Zibrov, P. R. Hemmer, and M. D. Lukin, Science 316, 1312 (2007).

[17] C. G. Yale, B. B. Buckley, D. J. Christle, G. Burkard, F. J. Heremans, L. C. Bassett, and D. D. Awschalom, Proc. Natl. Acad. Sci. U.S.A. 110, 7595 (2013).

[18] H. Kosaka, H. Shigyou, Y. Mitsumori, Y. Rikitake, H. Imamura, T. Kutsuwa, K. Arai, and K. Edamatsu, Phys. Rev. Lett. 100, 096602 (2008).

[19] H. Kosaka, T. Inagaki, Y. Rikitake, H. Imamura, Y. Mitsumori, and K. Edamatsu, Nature (London) 457, 702 (2009).

[20] P. Neumann, N. Mizuochi, F. Rempp, P. Hemmer, H. Watanabe, S. Yamasaki, V. Jacques, T. Gaebel, F. Jelezko, and J. Wrachtrup, Science 320, 1326 (2008).

[21] L. Childress, J. M. Taylor, A. S. Sorensen, and M. D. Lukin, Phys. Rev. Lett. 96, 070504 (2006).

[22] W. J. Munro, K. A. Harrison, A. M. Stephens, S. J. Devitt, and K. Nemoto, Nat. Photonics 4, 792 (2010).

[23] R. Raussendorf and H. J. A. Briegel, Phys. Rev. Lett. 86, 5188 (2001).

[24] N. Manson, J. Harrison, and M. Sellars, Phys. Rev. B 74, 104303 (2006).

[25] P. Tamarat et al., New J. Phys. 10, 045004 (2008).

[26] A. Batalov, V. Jacques, F. Kaiser, P. Siyushev, P. Neumann, L. Rogers, R. McMurtrie, N. Manson, F. Jelezko, and J. Wrachtrup, Phys. Rev. Lett. 102, 195506 (2009).

[27] K.-M. C. Fu, C. Santori, P. E. Barclay, L. J. Rogers, N. B. Manson, and R. G. Beausoleil, Phys. Rev. Lett. 103, 256404 (2009).

[28] M. W. Doherty, N. B. Manson, P. Delaney, and L. C. L. Hollenberg, New J. Phys. 13, 025019 (2011).

[29] J. R. Maze, A. Gali, E. Togan, Y. Chu, A. Trifonov, E. Kaxiras, and M. D. Lukin, New J. Phys. 13, 025025 (2011).

[30] T. Inagaki, H. Kosaka, Y. Mitsumori, and K. Edamatsu, Phys. Rev. B 89, 085311 (2014). 